\title{
Convergent Local Identity and Topographic Projection of Sensory Neurons
}

\author{
M. Rezaul Karim ${ }^{1,2}$ and Adrian W. Moore ${ }^{1}$ \\ ${ }^{1}$ Disease Mechanism Research Core, RIKEN Brain Science Institute, Wako, Saitama, 351-0198, Japan, and 2Graduate School of Science and Engineering, \\ Saitama University, Sakura-ku, Saitama, 338-8570, Japan
}

Development of sensory neural circuits requires concurrent specification of neuron modality, position, and topographic projections. However, little is understood about how controls over these distinct parameters can unify in a single developmental sequence. To address this question, we have used the nociceptive class IV dendritic arborization neurons in the Drosophila larval body wall as an excellent model that allows precise spatiotemporal dissection of developmental-genetic control over sensory neuron positioning and wiring, and subsequent analysis of its functional significance for sensorimotor behavior. The class IV neurogenetic program is intrinsic to the anterior domain of the embryonic parasegment epithelium. Along the ventrolateral axis of this domain, nociceptive neuron induction requirements depend upon location. Near the ventral midline, both Hedgehog and Epithelial growth factor receptor signaling are required for class IV neurogenesis. In addition, close to the ventral midline, class IV neurogenesis is preceded by expression of the Iroquois factor Mirror that promotes local nociceptive neuron differentiation. Remarkably, Mirror is also required for the proper routing of class IV topographic axonal projections across the midline of the CNS. Manipulation of Mirror activity in class IV neurons retargeted axonal projections and caused concordant changes in larval nociceptive escape behavior. These findings indicate that convergent sensory neuron specification, local differentiation, and topographic wiring are mediated by Mirror, and they suggest an integrated paradigm for position-sensitive neural development.

\section{Introduction}

Development of sensory neural circuits requires concurrent specification of neuron modality, position, and topographic projections. For example, the photoreceptors in the eye (Luo and Flanagan, 2007; Sanes and Zipursky, 2010), mechanoceptors in the whiskers (Erzurumlu et al., 2010), and nociceptors in the skin (Schouenborg, 2002) are present in large arrays of neurons with shared sensory modality. In each of these organs, individual sensory neurons must both properly detect a stimulus and accurately relay its position to the CNS.

Current models are limited in the spatiotemporal precision required to explain how controls over these distinct parameters unify in a single developmental sequence. To address this question, we have exploited the nociceptive class IV dendritic arborization neurons in the Drosophila larval body wall as an excellent model to examine developmental-genetic control over positioning and wiring of sensory neurons. In this model we are able to unambiguously and reproducibly identify every individual neural precursor, intermediate precursor (IP), and neuron during sensory system development.

\footnotetext{
Received June 6, 2011; revised 0ct. 1, 2011; accepted 0ct. 5, 2011.

Author contributions: M.R.K. and A.W.M. designed research; M.R.K. performed research; M.R.K. and A.W.M. analyzed data; M.R.K. and A.W.M. wrote the paper.

Funding was provided by RIKEN. Flybase (flybase.org) provided extensive information services. We thank Virginie Orgogozo, Charles Yokoyama, Tomomi Shimogori, Hokto Kazama, and Moore Laboratory members for discussions and encouragement, and Saori Akimoto for help with illustrations.

The authors declare no competing financial interests.

Correspondence should be addressed to Adrian W. Moore, Disease Mechanism Research Core, RIKEN Brain Science Institute, 2-1 Hirosawa, Wako, Saitama, 351-0198, Japan. E-mail: adrianm@brain.riken.jp.

DOI:10.1523/JNEUROSCI.2815-11.2011

Copyright $\odot 2011$ the authors $\quad 0270-6474 / 11 / 3117017-11 \$ 15.00 / 0$
}

Activation of class IV nociceptors causes the larva to initiate a stereotyped fast lateral rolling escape response, and the direction of rolling is tightly coupled to the location from which the noxious stimulus is received (Hwang et al., 2007). Control over neuron positioning may be significant for this escape response. Three class IV neurons are spaced equidistantly along the dorsoventral axis of each abdominal segment (Fig. $1 \mathrm{~A}$, blue diamonds) leading to a complete and nonoverlapping coverage of the body wall by class IV naked sensory dendritic endings (Grueber et al., 2003b). Class IV axons also show topographic mapping in the ventral nerve cord (VNC). Ventrally situated vdaB neurons send commissural axon branches over the midline of the VNC (Fig. 1 Bii). On the other hand, v'ada neurons situated more laterally do not (Fig. 1 Bi) (Grueber et al., 2007).

Class IV neurons are a subset of multidendritic (MD) neurons, all of which derive from the embryonic epithelium. In the embryonic epithelium, individual cells are specified as sensory organ precursor cells (SOPs). SOPs divide through an IP stage, and a single daughter of each SOP differentiates as an MD neuron (Fig. 1C). The position of SOP formation prefigures the class of neuron it will give rise to (Fig. 1D) (Orgogozo et al., 2001). Furthermore, mutations that cause SOP or IP duplication produce identical MD neurons (Moore et al., 2002, 2004; Grueber et al., 2003b; Soba et al., 2007). These findings suggest that potential for nociceptive class IV differentiation is encoded within the SOP lineage and dependent on lineage position.

Both class IV modality and topography show some relationship with neuron position in the body wall. vdaB and v'ada class IV neurons derive from the ventral embryonic epithelium, a clas- 


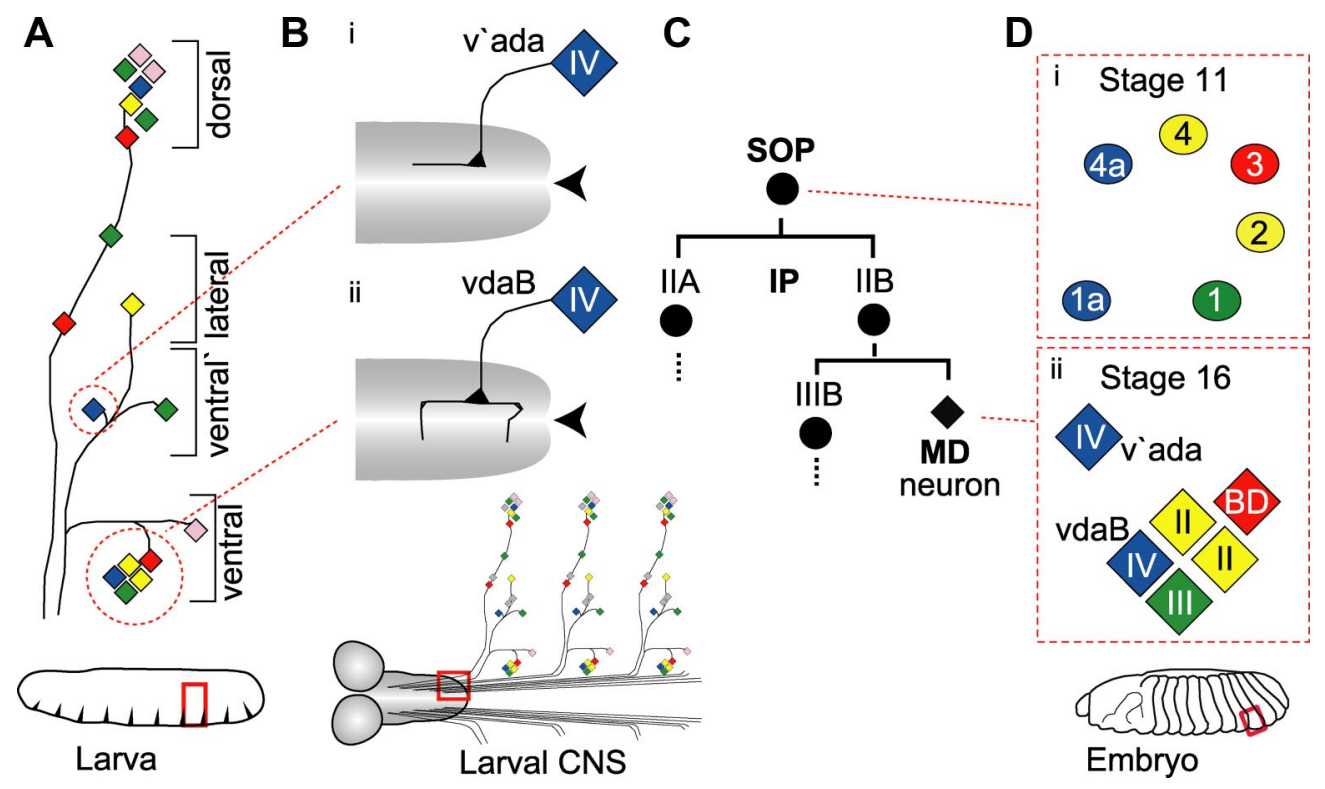

Figure 1. Organization and development of the Drosophila larval sensory PNS. A, A schematic showing the organization of MD neurons in abdominal segment 5 of the Drosophila larva (red box). Each MD class is color-coded: class I, pink; class II, yellow; class III, green; class IV, blue; BD, red. The circles highlight the ventral cluster of five MD neurons and one class IV neuron in the ventral prime region. $\boldsymbol{B}$, A schematic of the third instar larval CNS showing the axon termini of ventral prime class IV (v'ada) (Bi) and ventral class IV (vdaB) (Bii). The red box highlights where the axons enter and terminate in the VNC. This schematic is illustrative, not to scale. C, Each SOP-derived lineage produces a single MD neuron. IIA, IIB and IIIB are SOP-derived IPs. Di, In the embryo at st11, six SOP cells $(1 a-4 a)$ develop in stereotypic positions in the ventral embryonic epithelium (red box highlighted in the diagram of the embryo). The SOP cells are colored to represent the MD neuron class they will generate. Dii, At st16, six MD neurons form, one from each SOP cell. Neurons elaborated from SOPs 1, 2, 3, 4 and 1a form the ventral cluster. This consists of one class III neuron, two class II neurons, one bidirectional (vbd) neuron and one class IV neuron (vdaB). Class IV v'ada, derived from SOP 4a, migrates in a lateral direction to the ventral-prime region. All panels in all figures are orientated left, anterior; up, dorsal.

sic model in which to study hierarchical genetic control of epithelial patterning (Sanson, 2001). From this entry point, we investigate how mechanisms that position and wire the nociceptive class IV neurons unify in a single developmental sequence, and ask how these developmental mechanisms can shape function in the nociceptive-motor circuit.

\section{Materials and Methods}

Genetic crosses. For loss-of-function analyses we used $w g^{t s}\left(w g^{l-17} / w g^{l-12}\right)$, $E g f r^{t s}\left(E g f r^{t s l a} / E g f r^{24}\right), h h^{t s 2}, o d d^{5}, s l p 1^{05965}, k n^{1}, k n^{K N 2}$, mirr ${ }^{448}$ and mirr ${ }^{1825}$. act-lacZ or Dfd-GMR-YFP balancers were used to select the correct genotype. The mirr ${ }^{48} /$ mirr $^{1825}$ allelic combination survives at a low frequency to first instar larval stages, hence allowing time for the expression of $p p k-e G F P$, a late marker of class IV differentiation, to be initiated. For consistency in writing, in the text for any experiment, AEL (after egg laying) always refers to the time of development converted to the equivalent at $25^{\circ} \mathrm{C}$. For temperature-sensitive protein inactivation, embryos were collected for $30 \mathrm{~min}$ and raised at $18^{\circ} \mathrm{C}$ then temperatureshifted to $29^{\circ} \mathrm{C}$. Embryos were then switched back to $18^{\circ} \mathrm{C}$ and incubated until the correct stage for immunostaining. For heat-shock-mediated ectopic gene expression we used $h s-w g$, ( $h s$-Gal4, UAS- $\left.h h^{N}\right)$, or ( $h s-G a l 4$, $U A S$-spi). Embryos were collected for $30 \mathrm{~min}$ and aged at $25^{\circ} \mathrm{C}$, then heat-shocked at $37^{\circ} \mathrm{C}$ in a water bath for $20 \mathrm{~min}$, and then further cultured at $25^{\circ} \mathrm{C}$ until the stage required for analysis.

A sixmer repeat of an autoregulatory sc promoter E-box $(S c E 1)_{6}$ (kindly provided by A. Jarman, University of Edinburgh, UK) (Powell et al., 2004) was cloned into $P U A S T$ by standard protocols and then injected to make an SOP cell-specific $(S c E 1)_{6}$-Gal4 transgenic stock. For targeted gene expression in SOP cells, $(S c E 1)_{6}-$ Gal4 was crossed with UAS-mirr.

For targeted expression of dominant repressors, neur-Gal4 was crossed to either $U A S-c i^{R}$, or UAS-Axn.H, or UAS-Egfr.DN. For targeted mirr reduction-of-function, UAS-mirrRNAi was obtained from the Vienna Drosophila Rnai Center and recombined with mirr ${ }^{1825}$. Then neurGal4 was crossed with this mirr ${ }^{1825}$, UAS-mirrRNAi stock. Notably for both dominant repressor and RNAi experiments, use of the neur-Gal4 but not the $(\mathrm{ScE} 1)_{6}$-Gal4 driver produced a loss of neuron phenotype.
The differential activities of these drivers are likely due to the timing of their expression. neur-Gal4 is expressed in the epithelium initiating before SOP formation. ( $S C E 1)_{6}-$ Gal4 is expressed only after SOP formation.

To generate MARCM (mosaic analysis with a repressible cell marker) clones $w$; mirr ${ }^{e 48}$, FRT2A/TM3 Dfd-GMR-YFP or control $w$; FRT2A females were crossed to males from the MARCM driver stock $h s F L / y$; Gal4 ${ }^{109-2-80}$, UAS-mCD8::GFP; TubP-Gal80, FRT2A/SM5-TM6b. To generate stocks for flippase (FLP)-out clonal analysis, we crossed a recombinant (ScE1) ${ }_{\sigma}$-Gal4, ppk-Gal4 driver line to $y w, h s F L P$; UAS-FRTCD2,y+-stop-FRT-mCD8::GFP; UAS-mirr or control $y w$, hsFLP; UAS-FRT$C D 2, y+$-stop-FRT-mCD8::GFP flies. This driver combination allows the expression of Mirr from the SOP stage, and permits labeling of the differentiated MD neuron. For MARCM and FLP-out, heat shock, dendrite visualization and axon termini staining protocols were as previously described (Karim and Moore, 2011).

Drosophila stocks were kindly provided by Y.N. Jan (University of California, San Francisco, CA), T. Tabata (University of Tokyo, Japan), S. Hayashi (RIKEN Center for Developmental Biology, Kobe, Japan), V. Hatini (Tufts University, Boston, MA), H. McNeill (Samuel Lunenfeld Research Institute, Toronto, Canada), H. Krause (University of Toronto, Canada), L. Nilson (McGill University, Montreal, Canada), T. Uemura (University of Kyoto, Japan), Vienna Drosophila Rnai Center (Institute of Molecular Pathology, Vienna, Austria) and the Bloomington Drosophila Stock Center (Indiana University, Bloomington, IN).

Behavior. Behavioral analyses were performed as previously described (Hwang et al., 2007; Babcock et al., 2009). Embryos were collected for $2 \mathrm{~h}$ at $25^{\circ} \mathrm{C}$ and then cultured to third instar larva at $29^{\circ} \mathrm{C}$. Individual tests consisted of batches of 10 larvae, each batch from an independent collection of embryos (Hwang et al., 2007).

Immunohistochemistry, microscopy, and image analysis. Embryos and larvae were fixed and stained as per standard protocols. Figures of immunostainings or live imaging show a single or projection of several consecutive sections. Images were taken using Nikon C1, Leica SP2, or Leica TCS SP5 confocal microscopes, and then processed within guidelines using Photoshop and Illustrator (Adobe Systems Inc). Visualization of dendrite morphological characteristics was performed as described by 
Karim and Moore (2011), then analyzed by Neurolucida and Neuroexplorer software (MicroBrightField Inc.).

For immunohistochemistry, the antibodies used were as follows: rabbit anti- $\beta$-galactosidase, 1:2000 (MP Biomedicals); anti-GFP, 1:1000 (Invitrogen); anti-Ladybird Early, 1:2500 (K. Jagla, INSERM, ClermontFerrand, France); anti-Mirror, 1:1000 (H. McNeill, Samuel Lunenfeld Research Institute, Toronto, Canada); anti-Slp, 1:200 (East Asian Distribution Center for Segmentation Antibodies, National Institute of Genetics, Mishima, Japan); mouse anti- $\beta$-galactosidase, 1:200; anti-CD2, 1:700 (AbD Serotec); anti-Wingless, 1:10, anti-Cut, 1:100, and rat antiELAV, 1:200 (all Developmental Studies Hybridoma Bank, University of Iowa, Iowa City, IA); guinea pig anti-Odd, 1:200 (East Asian Distribution Center for Segmentation Antibodies, National Institute of Genetics, Mishima, Japan); anti-Knot, 1:1000, and anti-Senseless, 1:800 (H. Bellen, Baylor College of Medicine, Houston, TX). Fluorescently labeled secondary antibodies (Jackson ImmunoResearch Laboratories) were used at 1:300.

To measure $p p k-e G F P$ levels in different genetic backgrounds, each larva was anesthetized at first instar (Sugimura et al., 2003), or immobilized via pressure from the coverslip at third instar (Karim and Moore, 2011). $z$-stack images through the neuron cell body were taken of the live neuron using a Leica TCS SP 5 confocal microscope with a Plan APO CS $40.0 \times 1.25 \mathrm{NA}$ Oil lens. The settings were as follows: pinhole 1.67, digital zoom $4 \times, 512 \times 512$ pixels, $1.01 \mu \mathrm{m} z$-stack step, laser (458 $\mathrm{nm}$ Argon) at $12 \%$ power. Using ImageJ (National Institutes of Health), the cell body was highlighted as a 'region of interest' and the average pixel intensity in the $z$-section with maximum value was used (Grueber et al., 2003a). Background fluorescence levels were subtracted from this value as previously described (Grueber et al., 2003a).

\section{Results}

\section{Class IV neurogenesis occurs in epithelium with anterior identity}

In the ventral region of each parasegment, six SOP cells (1a, 1, 2, 3, 4, and 4a) form in invariant positions (Fig. 1Di). Class IV neurons derive from SOPs 1a and $4 \mathrm{a}$, which delaminate from the epithelium in the anterior region of the parasegment. Other classes derive from SOPs in the posterior region (Fig. 1 Dii). Anterior-posterior epithelial patterning in each parasegment is specified by a well described developmental cascade of transcription factors (TFs) (Nasiadka and Krause, 2002). We performed immunohistological analysis to relate the expression domains of these TFs to SOP positions. Together the combined activities of the zinc finger TF Odd skipped (Odd) and the Forkhead TF Sloppy-paired1 (Slp) cover all regions in ventral embryonic epithelium in which SOPs form. Slp expression overlapped SOPs 1, 2, 3, and 4 (Fig. 2 A). As previously described (Saulier-Le Dréan et al., 1998; Nasiadka and Krause, 2002), at an earlier time point, stage (st) 6, Odd was expressed adjacent and anterior to Slp, and posterior to the cells that prefigure the Hh-expression domain at the parasegmental boundary (PSB) (Fig. 2B). The Odd expression domain was lost before SOP formation. However, the class IV-generating SOPs 1a and 4a develop anterior to the Slp domain (Fig. 2A), and immediately posterior to but not overlapping the Hh expression domain (Fig. 2C). Therefore Odd expression prefigures the domain where SOPs 1a and 4a will arise.

We investigated whether odd and slp activity control class IV neurogenesis. We examined MD neuron identity in st16 embryos. To detect all neurons we used antibodies against Embryonic Lethal Abnormal Visual system (Elav) (Fig. 2D). In addition, to distinguish between MD neuron classes we used antibodies to detect class-specific markers. We labeled the class IVspecific Collier/Olf/Ebf family TF Knot (Kn) (Jinushi-Nakao et al., 2007) (Fig. 2D); the ventral BD (vbd)-specific Ladybird homeobox family TF Ladybird early (Lbe) (De Graeve et al., 2004) (Fig. 2D); and the atypical homeodomain TF Cut, which is absent in vbd, expressed at low levels in class II, intermediate levels in class IV, and high levels in class III neurons (Grueber et al., 2003a) (data not shown). In odd mutants anterior-derived class IV neurons were lost (vdaB, 50\% and v'ada, $50 \% ; n=44$ ) (Fig. $2 E$ ). On the other hand, the Lbe-positive vbd neuron was duplicated (39\%, $n=44)$ (Fig. $2 E$ ), along with extra class II and class III neurons ( $25 \%$ and $16 \%, n=32$, respectively, data not shown). In contrast st16 slp mutants showed duplication of both anteriorderived class IV neurons: ventral vdaB $(18 \%, n=23)$ and more lateral v'ada ( $14 \%, n=23)$, and concurrent loss of posteriorderived neuron classes (Fig. 2 F).

To investigate the underlying parasegmental alterations affecting class IV number, we examined the spatial organization of SOPs in odd and slp embryos. We mapped SOP position against $\mathrm{Wg}$ in the posterior, and $\mathrm{Hh}$ in the anterior, of the parasegment (Sanson, 2001). In the posterior of the parasegment SOPs 2 and 3 formed within the Wg expression domain, and SOPs 1 and 4 were positioned immediately anterior to this domain (Fig. $2 C$ ). In the anterior, SOPs 1a and 4a were immediately adjacent and posterior to the $\mathrm{Hh}$ expression domain (Fig. 2C). Using $\mathrm{Wg}$ as a marker, we next examined SOP formation in odd and slp mutant embryos. st 12 odd embryos showed a mirror-image duplication of the Wg expression domain and a concurrent replication of SOP cells within and adjacent to this ectopic domain (Fig. 2G). On the other hand, st12 slp embryos showed loss of SOP formation in the posterior, and duplicated lineages in the anterior, of the parasegment (Fig. $2 \mathrm{H}$ ).

Class IV formation is promoted by Odd and suppressed by Slp (summarized in Fig. 2I). Together, these data show that class IV neurogenesis occurs from SOPs that have anterior identity.

\section{Class IV induction requirements differ depending upon position along the ventrolateral axis}

What promotes class IV identity downstream of Odd? Both vertebrates and invertebrates use secreted morphogens to pattern neural precursors (O’Leary et al., 2007; Dasen, 2009; Erzurumlu et al., 2010). One function of Odd during epithelial patterning is to position the Hh expression domain at the PSB (Nasiadka and Krause, 2002). Hh in turn acts as a secreted morphogen to pattern the epithelium in the anterior of the parasegment (Sanson, 2001). As class IV-associated SOPs form immediately adjacent to the $\mathrm{Hh}$ expression domain (Fig. 2C), we investigated $\mathrm{Hh}$ function in class IV neurogenesis.

The Hh pathway is used in multiple developmental processes. Therefore, to avoid pleiotropic effects we used a temperaturesensitive $h h$ allele and used temperature-shifts from 18 to $29^{\circ} \mathrm{C}$ to disrupt pathway activity at consecutive stages of MD neurogenesis (Fig. 3A). Disrupting pathway activity at any stage after SOP formation gave no alteration in MD number or TF expression (Fig. 3A, black bars). On the other hand, disrupting Hh pathway activity before SOP formation caused loss of MD neurons (Fig. $3 A$, green or orange bars). We report data for the latest stage at which strong phenotypes were caused-heat inactivation between 4.5 and $5.5 \mathrm{~h}$ AEL (Fig. 3A, orange bar).

In st 16 hh embryos, the ventral class IV neuron vdaB was lost in $20 \%$ of parasegments $(n=91)$ (Fig. $3 C)$. This loss of vdaB was preceded by SOP1a loss $(23 \%, n=47)$ (Fig. $3 D)$. On the contrary, there was no loss of v'ada $(n=91)$ or SOP4a $(n=47)$. In $h h$ mutants we also noted that other non-class IV MD neurons were lost in the ventral cluster, for example vbd was lost in $62 \%$ of embryos $(n=91)$ (Fig. 3C). We hypothesized that the loss of posterior-derived MD neurons may be due to a secondary dis- 


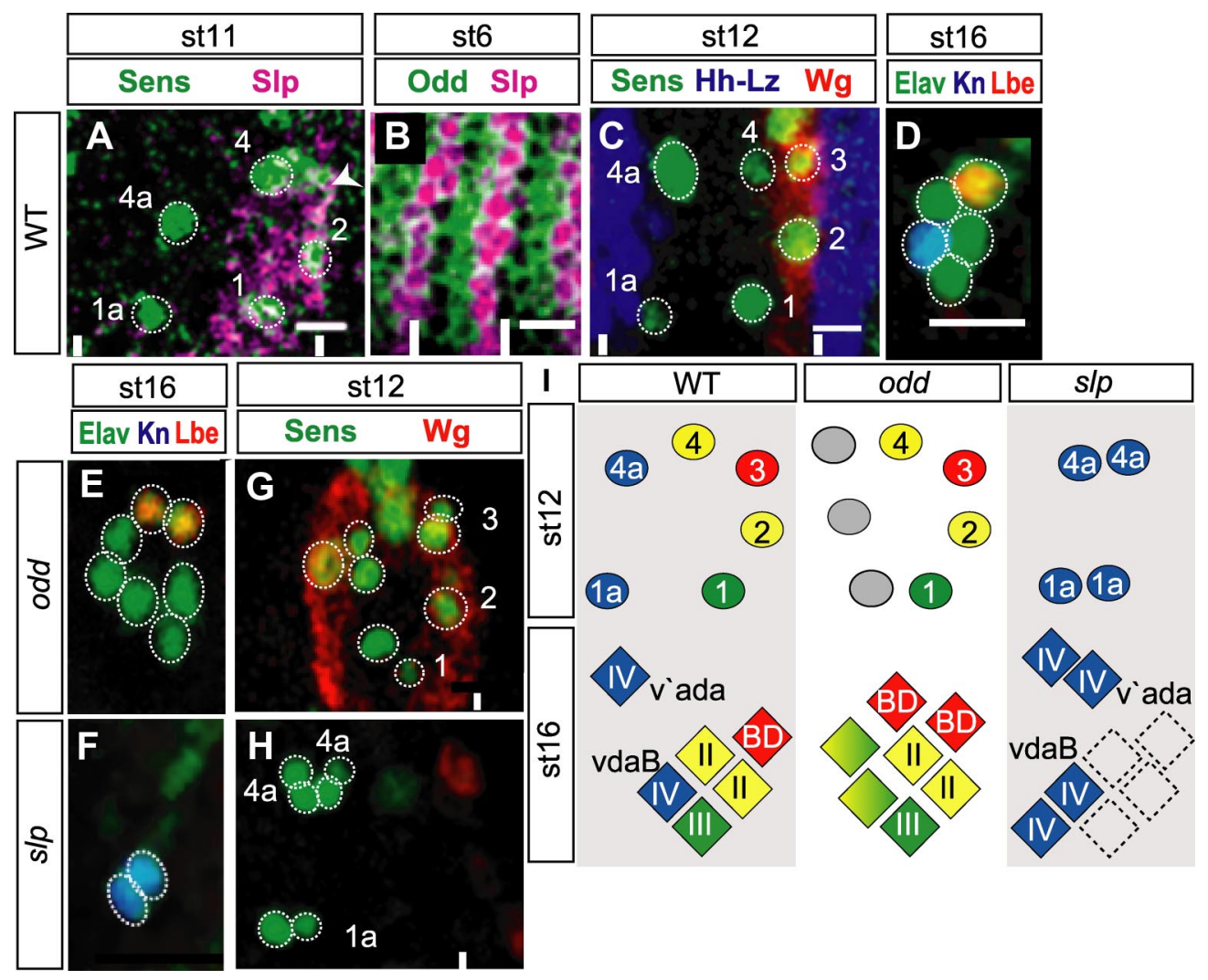

Figure 2. Control of anterior-posterior epithelial identity via $0 \mathrm{dd}$ and SIp is upstream of class IV neuron formation. $\boldsymbol{A}$, Ventral embryonic epithelium of st11 embryos stained with fluorescent antibodies to detect Sens (green), and SIp (magenta). Note 1: Not all of SOP1a-4a form at the same time. In this panel an arrowhead indicates the approximate region where SOP3 will form. $\boldsymbol{B}$, Future ventral epithelium of a st6 embryo after transition from a 7 to 14 stripe pattern, it is stained with fluorescent antibodies to detect Odd (green), and Slp (magenta). Each forming parasegment consists of a posterior Slp-expressing domain. Anterior and adjacent to this is an Odd-expressing domain. Anterior to the Odd-expressing domain is an unlabeled domain that represents the (Engrailed) domain where Hedgehog expression is induced (Saulier-Le Dréan et al., 1998; Nasiadka and Krause, 2002). Background staining in the green channel marks the cell membrane and so indicates the position of the cell boundaries. C, Ventral embryonic epithelium of a st12 embryo stained with fluorescent antibodies to detect Sens (green), Wg (red), and $\beta$-galactosidase to detect $h h$-lacZ expression (blue). D, Ventral MD neuron cluster of a WT st16 embryo, labeled to detect Elav (green), Kn (blue), and Lbe (red). $\boldsymbol{E}$, st16 odd embryos, showing an increase in posterior-derived MD neurons. $\boldsymbol{F}$, st $16 \mathrm{~s} / \mathrm{p}$ embryos have duplications of anterior-derived class IV neurons, and concurrent loss of posterior-derived neurons. Note 2: In both odd and s/p embryos alternate parasegments have a stronger phenotype reflecting the double-segment periodicity bias of the activity of these factors. The quantifications reported in the results section are the average across all parasegments. G, st12 odd embryos labeled to detect Sens (green) and Wg (red). There is a mirror-image duplication of the Wg expression domain and a concurrent duplication of the SOP cells in this duplicated domain. Note 3: In our figures throughout this study, at each SOP position where we saw anti-Sens staining we detected either a single cell (the SOP, e.g., position 2 in G) or its immediate daughter cells (the lla and Illb cells, e.g., position 3 in $\mathbf{G}$. $\boldsymbol{H}$, st12 s/p embryos show loss of posterior and duplication of anterior SOPs. $\boldsymbol{I}$, Diagrams summarizing how the control of body wall identity by odd and s/p prefigures MD neuron identity; color-coding as in Figure 1. Scale bars, $10 \mu \mathrm{m}$. Vertical bars, The parasegment boundaries.

ruption in Wg activity because at st11-12 Hh activity maintains Wg expression at the PSB (Fig. 3D) (Ingham and Hidalgo, 1993).

To test this, we also examined the role of $\mathrm{Wg}$. Indeed $w g^{t s}$ embryos showed loss of neurons derived from SOPs in the posterior of the parasegment: the vbd neuron was lost in 100\% $(n=$ $72)$, the class III lost in $85 \%(n=44)$ and class II neurons in $73 \%$ $(n=44)$ of parasegments (Fig. $3 E)$. On the contrary, in $w g$ mutants both class IV neurons, vdaB and v'ada, always formed $(n=$ 72 ). MD neuron loss in $w g$ mutants was always preceded by the loss of posterior SOPs: all four SOPs were lost in 45\%, three were lost in 35\%, and two lost in $20 \%$ of parasegments $(n=71)$ (Fig. $3 F$ ). These data suggest that $\mathrm{Hh}$ has a direct effect on $\mathrm{vdaB}$, and only indirectly effects non-class IV neurons through failure to maintain Wg levels.

To confirm a direct relationship between $\mathrm{Hh}$ and vdaB formation we inactivated only one copy of $h h(h h /+)$. This manipulation did not lead to observable reductions in Wg expression (Fig. $3 H$ ), and no loss of MD neurons other than class IV occurred (Fig. $3 F, G$ ). Nonetheless the vdaB neuron, but not the v'ada, was lost in $17 \%$ of parasegments $(n=35)$ (Fig. $3 B, G)$. Furthermore, SOP1a was lost $(15 \%, n=40)$ (Fig. $3 H$ ); however, SOP4a was never lost.
Because class IV induction requirements via Hh differed depending upon location along the ventrolateral axis, we also investigated the role of Egfr adjacent to the midline (Urban et al., 2004). In st16 Egfr embryos the two neurons that normally arise closest to the midline were lost, $\operatorname{vdaB}(79 \%, n=70)$ (Fig. 3I) and the class III $(76 \%, n=29)$. Loss of these neurons was preceded by the loss of SOPs 1a (73\%) and $1(69 \%, n=41)$ (Fig. 3J).

To complement these temperature-sensitive analyses, we also performed dominant repressor experiments. neur-Gal4 was used to drive, either a repressor form of Cubitus interruptus $\left(\mathrm{Ci}^{\mathrm{R}}\right)$ (Hepker et al., 1997), Axin (Hamada et al., 1999), or dominantnegative Egfr (Egfr ${ }^{\mathrm{DN}}$ ) (Buff et al., 1998) in the ventral embryonic epithelium before SOP formation. Expression of these factors represses the intrinsic ability of cells to respond to Hedgehog, Wingless, or Egfr signaling, respectively. In neur-Gal4, UAS-ci ${ }^{R}$ st 16 embryos, the ventral vdaB class IV neuron was lost in $24 \%$ of parasegments; however, the more lateral v'ada was never lost. Posterior derived MD neurons were also lost, e.g., vbd was lost in $75 \%$ of parasegments $(n=59)$. In neur-Gal4, UAS-Axn.H embryos posterior-derived MD neurons were lost, e.g., vbd (99\%). On the other hand, vdaB and v'ada were never lost $(n=55)$. In neur-Gal4, UAS-Egfr ${ }^{D N}$ embryos, vdaB was lost in $75 \%$ of para- 

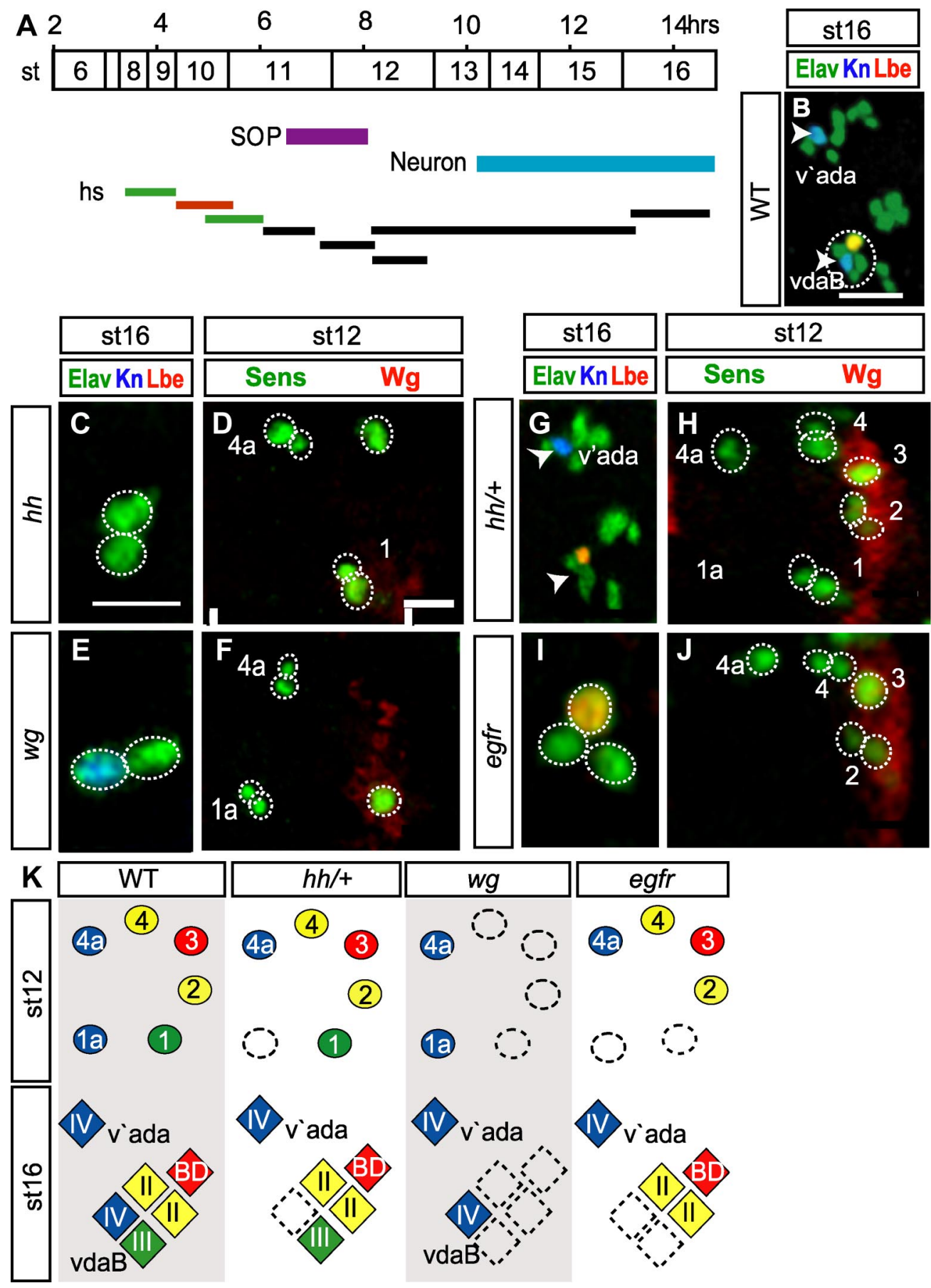

Figure 3. $\mathrm{Hh}, \mathrm{Wg}$, and Egfr are required for local MD neuron formation. A, Timeline of MD neuron development, highlighted stages: SOP, purple; and MD neuron, blue. Bars represent the periods in which $\mathrm{Hh}, \mathrm{Wg}$, and Egfr activity was disrupted by temperature-shift. The orange bar shows the latest point at which inactivation of $h h, w g$, and Egfr gave a strong $M D$ neuron number phenotype. All heat shock inactivation data reported in the results and shown in this figure are from inactivation at this time point $\left(4.5-5.5 \mathrm{~h} \mathrm{AEL}\right.$ at $\left.25^{\circ}\right)$. B, $\boldsymbol{C}, \boldsymbol{E}, \mathbf{G}, \mathbf{I}$, st 16 embryos labeled with antibodies to detect Elav (green), Kn (blue), and Lbe (red). $\boldsymbol{B}$ and $\mathbf{G}$ show both ventral and ventral prime clusters. Arrowheads indicate the position occupied by Kn-positive neurons: vdaB in the ventral cluster (circled in $\boldsymbol{B}$ ) and v'ada in the ventral prime cluster. $\boldsymbol{D}, \boldsymbol{F}, \boldsymbol{H}, \boldsymbol{J}$, Ventral embryonic epithelium of st 12 embryos stained with fluorescent antibodies to detect Sens (green), Wg (red). Vertical bars, The parasegment boundaries. $\boldsymbol{C}, \boldsymbol{D}, h h ; \boldsymbol{E}, \boldsymbol{F}, w g ; \boldsymbol{G}, \boldsymbol{H}, h h /+, \boldsymbol{I}, \boldsymbol{J}$, Egfr embryos; st16. $\boldsymbol{K}$, Diagrams summarizing the $h h$, wg, and Egfr loss-of-function phenotype, color-coded as in Figure 1. Hh, Wg, and, Egfr are required for localized SOP formation. All scale bars, $10 \mu \mathrm{m}$. Vertical bars, The parasegment boundaries.

segments. On the contrary, there was no loss of v'ada $(n=45)$. Examination of st12 embryos revealed that vdaB-loss was preceded by SOP1a-loss in neur-Gal4, UAS-ci ${ }^{R}$ embryos $(22 \%, n=$ $52)$ and neur-Gal4, UAS-Egfr $r^{D N}$ embryos $(78 \%, n=50)$. No SOP1a-loss occurred in neur-Gal4, UAS-Axn.H embryos $(n=$ 46), and SOP4a-loss never occurred in any of these treatments.

Together, these data demonstrate that class IV induction requirements differ along the ventrolateral axis. Close to the mid- line, vdaB requires both Hh and Egfr activity; further away, v'ada requires neither (summarized in Fig. $3 K$ ).

In the above disruption-of-function experiments, we saw no evidence of TF expression fate changes in the remaining neurons following the disruption of the $\mathrm{Hh}, \mathrm{Wg}$, and Egfr pathways. However, our manipulations caused a loss of SOP formation, and therefore did not fully preclude that these pathways could control fate via action within the SOP itself. To circumnavigate this prob- 


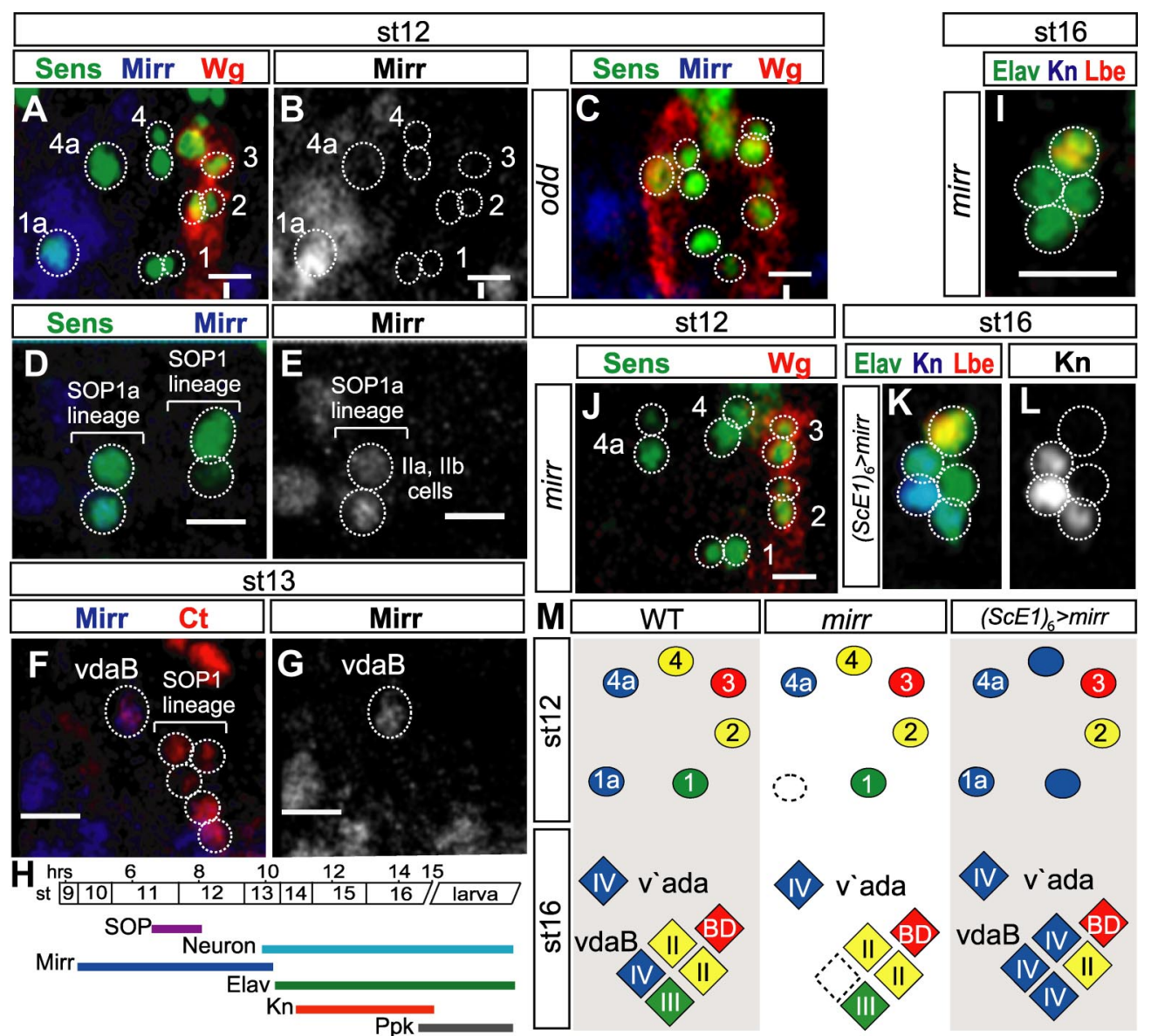

Figure 4. Mirr is required for class IV vdaB neuron formation. $\boldsymbol{A}-\boldsymbol{C}$, The ventral embryonic epithelium of st12 embryos, labeled to detect Sens (green), Wg (red), and Mirr (blue). $\boldsymbol{A}$, The Mirr expression domain overlaps with SOP1a. B, Mirr expression from $\boldsymbol{A}$. C, The ventral embryonic epithelium of a st12 odd mutant (segment as in Fig. 2G), in each alternate parasegment the Wg domain is duplicated and the anterior Mirr expression domain is concomitantly reduced or lost. Vertical bar, The parasegment boundary. $\boldsymbol{D}$, The ventral embryonic epithelium of st12 WT embryos, labeled to detect Sens (green), and Mirr (blue). Mirr continues to express in the SOP1a lineage Ila and Ilb IP cells. E, Mirr expression from D. F, st13 WT embryos labeled to detect Mirr (blue) and Cut (red) in the SOP lineage. Mirr is expressed in the nascent vdaB neuron. G, Mirr expression from $F$. $\boldsymbol{H}$, A Timeline of MD neurogenesis color-coded as in Figure $3 A$, showing in addition the temporal expression profiles of Mirr (dark blue), Elav (green), Knot (red), and Ppk (black). I, Ventral cluster of st16 mirr mutant embryos labeled to detect Elav (green), Kn (blue), and Lbe (red). vdaB is not formed. J, st12 mirr mutant embryos labeled to detect Sens (green) and Wg (red). SOP1a is not formed. $\boldsymbol{K}$, Ventral cluster of st16 (SCE1) ${ }_{6}$ Gal4, UAS-mirr embryos labeled to detect Elav (green), Kn (blue), and Lbe (red). $\boldsymbol{L}$, Kn expression from $\boldsymbol{K} ; 3$ neurons express $\mathrm{Kn} . \boldsymbol{M}$, Diagrams summarizing the mirr loss-of-function and gain-of-function phenotypes. Mirr promotes SOP1a and subsequent vdaB formation. Mirr can induce Kn expression in other SOP lineages. Scale bars, $10 \mu \mathrm{m}$.

lem, we complemented our studies with a gain-of-function approach in which SOPs were not lost. We used $h s$-wg or $h s-G a l 4$, $U A S-h h^{N}$ or $h s$-Gal4, UAS-spi (the Egfr ligand) at st11 during SOP formation to drive ubiquitous ectopic expression of $\mathrm{Wg}$ $(n=42), \mathrm{Hh}^{\mathrm{N}}(n=54)$, or Spi $(n=26)$ respectively. These manipulations changed epithelial cell fates hence altering denticle belt patterning (data not shown) but they did not alter MD neuron number or identity.

\section{Mirror is a localized ventral promoter of class \\ IV neurogenesis}

TF-mediated prepatterning of precursors drives neuron diversification in many systems (O'Leary et al., 2007; Dasen, 2009; Erzurumlu et al., 2010). In the absence of direct morphogen control over class IV fate, we searched for putative prepatterning TFs with expression domains exclusive to class IV SOPs. We examined a set of candidate TFs differentially expressed among Drosophila brain neuroblasts (Urbach and Technau, 2003) and identified the Iroquois (Irx) factor Mirror (Mirr).

Anti-Mirr immunolabeling demonstrated Mirr expression adjacent to the ventral midline. The Mirr expression domain spanned from the PSB through the anterior of the parasegment where it overlapped SOP1a. However, the Mirr expression domain did not extend into the posterior of the parasegment (Fig. $4 A, B)$. The Mirr expression domain in the anterior of the parasegment was reduced or lost in odd mutants (Fig. 4C), but not changed in heat-shock-inactivated $h h$ or Egfr embryos (data not shown). As the SOP1a lineage elaborated, Mirr remained expressed in the IPs but was lost from the surrounding epithelium (Fig. $4 D, E$ ). Mirr was expressed in the nascent vdaB neuron (Fig. $4 F, G)$. However, it was lost from this cell before differentiation as we were unable to detect costaining of Mirr and Elav or Kn (summarized in Fig. 4H). Mirr was never expressed in the v'ada lineage.

In st16 mirr mutants the vdaB neuron was lost with 34\% frequency $(n=35)$ (Fig. $4 I)$, and this was preceded by SOP1a loss $(35 \%, n=43)$ (Fig. 4J). However, all other ventral cluster MD neurons, as well as v'ada, were never lost. As mirr mutants had a loss-of-SOP phenotype, we assayed whether Mirr is instructive for class IV differentiation via a gain-of-function approach. We used an SOP-specific ( $\mathrm{SCE} 1)_{6}$-Gal4 transgene (Materials and Methods) to drive UAS-mirr in all SOPs. This caused a fate transformation of ventral cluster class II and III neurons, causing an increase of Kn- 


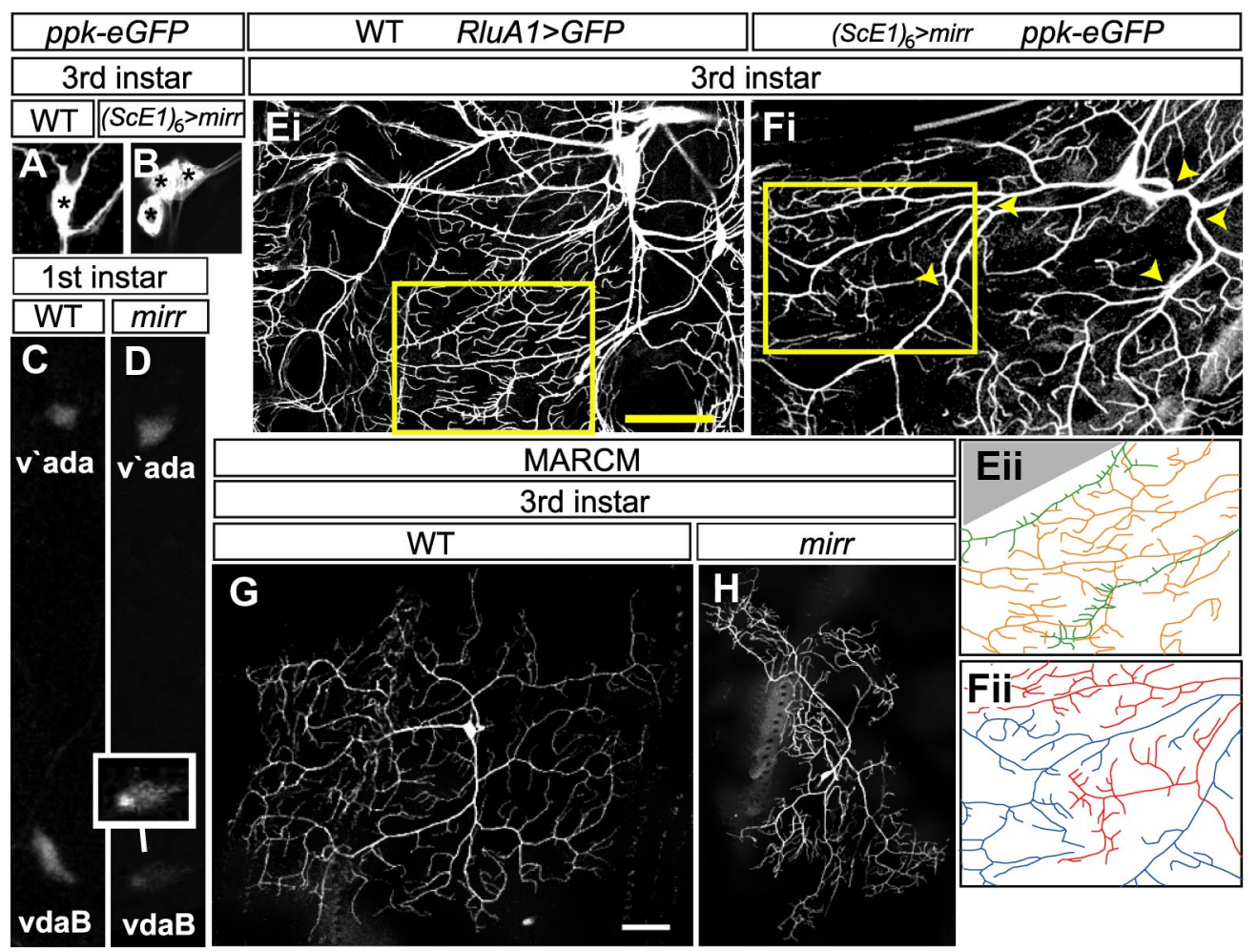

Figure 5. Mirr is required for vdaB class IV-specific differentiation. $\boldsymbol{A}, \boldsymbol{B}$, WT $(\boldsymbol{A})$ and $(S C E 1)_{6}$-Gal4, UAS-mirr, ppk-eGFP $(\boldsymbol{B})$ third instar larvae stained with an anti-GFP antibody. WT larvae have one ppk-eGFP-positive class IV neuron in the ventral cluster. (SCE1) ${ }_{6}$-Gal4, UAS-mirr larvae have 2 or 3 ppk-eGFP-positive neurons. Asterisks mark the individual cell bodies. C, D, WT (C) and mirr mutant (D) first instar larvae showing ppk-eGFP expression in ventral (vdaB) and ventral prime (v'ada) class IV neurons. To highlight vdaB, the inset neuron in $\boldsymbol{D}$ has the fluorescence levels in this neuron accentuated via image processing software. Ei, The ventral cluster of a WT third instar larva where all MD neurons and their dendritic arbors are marked via RIuAT-Gal4, UAS-mCD8::GFP (Jinushi-Nakao et al., 2007). Eii, A tracing of dendrite branches in the area highlighted with a yellow box in Ei, class IV dendrites are colored orange and class III green. Extensive overlap of the dendritic termini of these different neuron classes occurs. Fi, The ventral cluster of a $(S C E 1)_{6}$-Gal4, UAS-mirr, ppk-eGFP larva. The vdaB and a second transformed neuron both express ppk-eGFP that marks their dendritic arbors. The major trunks of these neighboring dendrite arbors overlap (yellow arrowheads). Fii, A tracing of the area highlighted with a yellow box in $F i$, The dendrite arbor is colored red for one neuron and blue for the other. The dendritic termini of these two neurons do not overlap, instead they partition the body wall into small domains. $\mathbf{G}, \boldsymbol{H}$, WT $(\mathbf{G})$ and mirr MARCM $\operatorname{vdaB}(\boldsymbol{H})$ clones in a third instar larva. mirr mutants show a failure to properly arborize over the normal dendritic field area. Scale bars, $35 \mu \mathrm{m}$.

positive MD neurons to $2(56 \%)$ or $3(17 \%)$ without changing the total number of neurons in the cluster $(n=118)$ (Fig. $4 K, L)$.

Mirr is expressed in the lineage that gives rise to the class IV $\mathrm{vdaB}$, and it is required for $\mathrm{vdaB}$ formation. Mirr is not expressed in the class IV v'ada lineage, and this neuron forms normally in mirr mutants. This finding highlights the differing requirements for the formation of these two class IV neurons. Furthermore, Mirr expression at SOP stages converts other lineages to produce neurons with class IV character (summarized in Fig. $4 M$ ).

\section{Mirror is a localized ventral promoter of class}

IV differentiation

Intriguingly Mirr is only expressed in the vdaB lineage, but it promotes class IV formation. To explain this puzzling result, we hypothesized that Mirr may act as a localized promoter of class IV differentiation close to the ventral midline.

To test this hypothesis, we first examined expression of pickpocket ( $p p k$ ), a hallmark ion channel for class IV neurons (Grueber et al., 2003b). In (ScE1) ${ }_{6}$-Gal4, ppk-eGFP, UAS-mirr larvae, the number of $p p k$-expressing neurons in the ventral cluster increased from 1 (vdaB) to $2(53 \%)$ or $3(15 \%)(n=104)$ (Fig. $5 A, B)$. In class IV neurons ppk expression is dependent on Kn activity (Hattori et al., 2007; Jinushi-Nakao et al., 2007; Crozatier and Vincent, 2008). We therefore addressed whether Mirr induced $p p k$ via a $\mathrm{Kn}$-dependent pathway. In a hypomorphic $k n$ background (Jinushi-Nakao et al., 2007) $\left(k n^{1} / k^{\mathrm{KN} 2} ;(S c E 1)_{6-G a l 4, ~ p p k-e G F P,}\right.$ UAS-mirr) both vdaB and neighboring transformed MD neurons had strongly reduced $p p k-e G F P$ expression. In $k n$ mutant vdaB neurons $(n=11) p p k-e G F P$ expression was reduced to $41 \%$ of wild-type (WT) $(n=11)\left(p=1.6 \times 10^{-5}\right.$, Student's two-tailed $t$ test $)$. Similarly, in $k n$ mutant transformed-MD neurons $(n=11)$ ppk-eGFP expression was reduced to only $8 \%$ the expression in a non- $k n$ mutant background $(n=11)\left(p=2.6 \times 10^{-13}\right.$, Student's two-tailed $t$ test).

In mirr mutants a subset of $\mathrm{vdaB}$ neurons are still formed. Therefore by examining these neurons we were able to test whether Mirr may also act as a localized promoter of class IV differentiation. To test whether Mirr is required for $p p k$ expression in $\mathrm{vdaB}$, we examined the level of $p p k-e G F P$ expression in mirr mutants (Materials and Methods, Fig. 5C,D). In WT early first instar larvae the ratio of $p p k$-eGFP expression is similar between vdaB and v'ada, $98 \% \pm 0.06$ (mean \pm SEM) $(n=17)$. On the other hand in mirr mutants the ratio of $p p k$-eGFP fluorescence between vdaB and v'ada within the same segment was significantly reduced to on average $69 \% \pm 0.06(n=19, p=0.0018$, Student's two-tailed $t$ test) (Fig. $5 C, D$ ). Together, the above data suggest that Mirr is required for proper induction of $p p k$ in the $\mathrm{vdaB}$ lineage, and this works through the previously described $k n$-dependent pathway (Hattori et al., 2007; Jinushi-Nakao et al., 2007; Crozatier and Vincent, 2008).

In conjunction with class-specific regulation of ion channel content, class IV neurons have a characteristic dendritic arbor, in which extensive dendrite branches evenly fill the body wall. The dendritic territories of neighboring class IV neurons do not overlap (Grueber 
et al., 2003b; Sugimura et al., 2003; Grueber and Sagasti, 2010). In contrast, the dendritic branches and territories of different MD classes frequently overlap, e.g., the branches of class III and class IV neurons (Fig. 5Ei,ii) (Grueber et al., 2003b; Sugimura et al., 2003; Grueber and Sagasti, 2010). In (ScE1) ${ }_{6}$-Gal4, ppk-eGFP, UAS-mirr larvae, additional $p p k$-positive neurons are formed by transformation of the ventral class III and class II lineages. These additionally formed class IV neurons lie adjacent to $\mathrm{vdaB}$, and we investigated the interaction between the dendrites of these neurons (Fig. 5B,Fi). The main trunks of the normal and transformed class IV neurons extensively overlapped (Fig. 5Fi, yellow arrowheads). On the other hand, their terminal branches partitioned the body wall into small domains without overlap $(100 \%, n=32$, Fig. 5Fii). These data indicate that ectopic mirr induces a class IV-identity dendritic repulsion program in the terminal branches of the transformed neurons.

Next we examined dendritic arbor characteristics of individual vdaB neurons in WT and mirr mutant MARCM clones (Karim and Moore, 2011). In mirr mutant vdaB neurons, dendritic field coverage (Sugimura et al., 2004) was significantly reduced to $84 \%$ of $\mathrm{WT}$, and branches failed to fully fill the body wall (Fig. 5G,H) (mirr: 143,984 $\mathrm{\mu m}^{2} \pm 7870$ (mean \pm SEM), $n=16$; WT: $170,850 \mu \mathrm{m}^{2} \pm 3490$, $n=16 ; p=0.0083$, Student's two-tailed $t$ test). This was not associated with a change in dendritic self-avoidance (Long et al., 2009) (mirr: 2.74 crossing points/ $1000 \mu \mathrm{m}$ dendritic length \pm 0.36 (mean \pm SEM), $n=12$; WT: $2.58 \pm 0.34$, $n=10 ; p=0.76$ Student's two-tailed $t$ test). However, it was correlated with a similar reduction of dendritic branching to $86 \%$ of WT (mirr: 380 dendrite ends \pm 20 (mean \pm SEM), $n=12$; WT: $443 \pm 10, n=$ $10 ; p=0.026$, Student's two-tailed $t$ test).

On the contrary, Mirr is not expressed in the v'ada lineage. As expected, there was no effect of mirr mutation on v'ada differentiation. Dendritic field coverage was unchanged (mirr: 232,398 $\mu \mathrm{m}^{2} \pm 5356$ (mean \pm SEM), $n=10$; WT: $241,274 \mu \mathrm{m}^{2} \pm 7608$, $n=10 ; p=0.34$, Student's two-tailed $t$ test), as was branching (mirr: 480 dendrite ends \pm 15 (mean \pm SEM), $n=12$; WT: $490 \pm$ $19, n=10 ; p=0.68$, Student's two-tailed $t$ test).

Together, these data suggest that Mirr acts as a localized ventral prepatterning factor that enables SOP 1a formation, and then promotes class IV character in the vdaB lineage. However, Mirr has no function in the more lateral v'ada lineage.

\section{Mirror directs topographic axonal projections}

$\mathrm{vdaB}$ and v'ada neurons have shared modality. However, they relay different spatial representations of the external world to the CNS via topographic axonal projections (Grueber et al., 2007) (Fig. $1 B$ ). Because of the differential expression of Mirr between the vdaB and v'ada lineages (Fig. $4 A, B, D-G$ ), we investigated Mirr as a candidate to control the ventral-specific topography of the vdaB axon.

WT vdaB neurons sent both anterior and posterior branches across the VNC midline $(n=9)$ (Fig. $6 A)$. On the other hand, in mirr MARCM clones midline crossing of the axon terminal branches was lost. In 19\% of mirr vdaB neurons both branches failed to cross the midline (Fig. $6 \mathrm{~B}$ ), and in a further $37 \%$ only the posterior branch crossed $(n=16)$. Hence Mirr activity is required for midline crossing by the axon termini of vdaB.

Is Mirr also instructive for midline crossing? To test this, we examined the effect of ectopic Mirr expression on the terminal axon branches of v'ada. $100 \%$ of WT v'ada neurons did not send terminal axon branches across the midline $(n=8)$ (Fig. 6C,D). Via a flp-out approach (Materials and Methods; Karim and Moore, 2011), we expressed Mirr in all SOP lineages, labeled all class IV axon termini with CD2, and simultaneously generated single v'ada mC8::GFP-positive clones. Ectopic Mirr in the v'ada lineage strongly induced midline crossing by the posterior branch of the v'ada axon terminal $(84 \%, n=19)$ (Fig. $6 E, F)$, and $62 \%$ of 

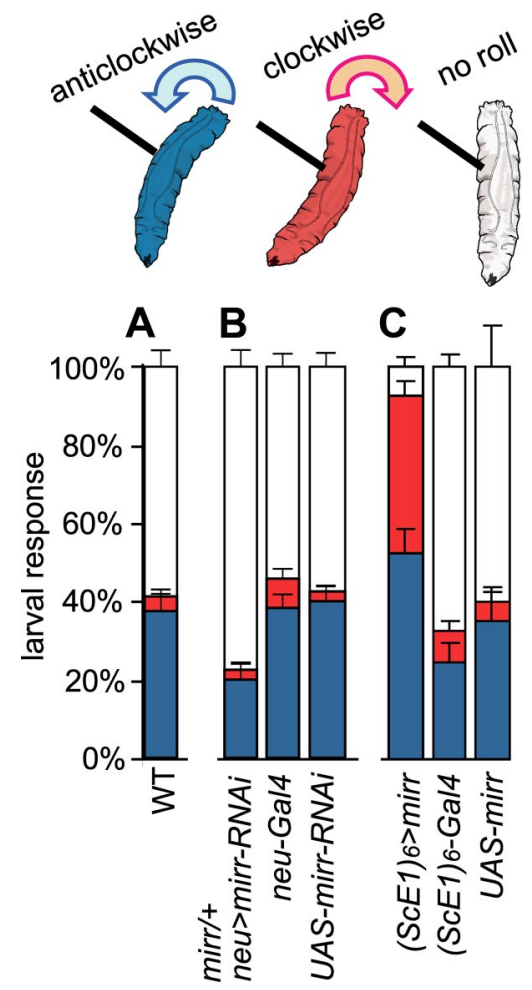

Figure 7. Class IV sensory system organization through Mirr shapes a nociceptive response. $A-C$, Bar charts indicating the percentage of animals that elicit a rolling response within a $20 \mathrm{~S}$ period. WT $(\boldsymbol{A})$, mirr reduction of function $(\boldsymbol{B})$, and ectopic expression of mirr $(\boldsymbol{C})$ in the SOP lineages. C, Ectopic expression of mirr in the SOP lineages leads to a greatly increased sensitivity to a noxious thermal probe applied to the lateral body wall. Furthermore, in WT the initiation of the rolling response is in an anticlockwise direction (blue); however, in manipulated larva the response is often initiated in a clockwise direction (red).

these altered axon termini had additional small terminal ramifications projecting toward the midline.

These data demonstrate that Mirr promotes midline crossing by the axon terminals. In particular, the difference in Mirr activity between the ventral vdaB and more lateral v'ada lineages controls the topographic projections of the resultant class IV axon termini.

\section{Manipulation of circuit architecture tunes a nociceptive response}

Our data identify Mirr as a genetic node linking specification of neuron modality, position, and topographic projections into one unified developmental sequence. Mirr hence shapes the architecture of the mature nociceptive sensory nervous system. Meticulous local control over this architecture suggests that it is important to delineate behavior. We therefore asked whether alterations in Mirr activity during circuit formation could produce alterations in larval nociceptive behavior.

We attempted to initiate a nociceptive escape response in third instar larvae by gently touching a hot probe to the lateral body wall of an abdominal segment (Babcock et al., 2009). The percentage of animals at a threshold temperature of $40^{\circ} \mathrm{C}$ that elicit a rolling response within a $20 \mathrm{~s}$ period indicates the degree of sensitivity to noxious stimulation (Babcock et al., 2009). In agreement with these previous studies, WT (Oregon R) larvae elicited a rolling response in $41 \%$ of trials ( \pm 3 SEM) (Fig. $7 A$ ), thus demonstrating this as a sensitized approach for examining alterations in nociceptive behavior.

To investigate whether Mirr activity functions in defining rolling behavior, we expressed a mirr-RNAi construct in the neuroepithelium of mirr heterozygotes (neur-Gal4/mirr, UASmirrRNAi) (Materials and Methods). In st16 embryos with this genotype, class IV vdaB loss occurred in $18 \%$ of cases $(n=79)$, and no other neuron loss was seen. In third instar larvae this manipulation lead to a reduction in the frequency at which the larvae initiated a rolling response to $22 \%$ ( \pm 4 SEM), compared with $42 \%$ ( \pm 4 SEM) or $46 \%$ ( \pm 4 SEM) in UAS-mirrRNAi/+ or neur-Gal4/+ controls, respectively (Fig. $7 B$ ). These data suggest a role for Mirr activity in defining the rolling response.

To focus Mirr manipulation solely in the SOP lineages, we turned to a targeted gain-of-function approach using $(S c E 1)_{6}-$ Gal4 to direct ectopic mirr expression ( $(\mathrm{ScE} 1)_{6}$-Gal4, ppk-eGFP, $U A S$-mirr $)$. Control larvae elicited a rolling response in $40 \%( \pm 8$ SEM, UAS-mirr + ) or $33 \%$ ( \pm 6 SEM, (ScE1) $)_{6}$ Gal4, ppk$e G F P /+)$ of trials. Furthermore, ectopic mirr-expressing larvae initiated and executed the rolling escape response with apparently normal muscle coordination. However, they exhibited a greatly heightened sensitivity to noxious stimulation, and initiated a roll in 93\% ( \pm 3 SEM) of trials (Fig. 7C). Furthermore, as previously described (Hwang et al., 2007), control larvae predominantly rolled in an anticlockwise direction relative to stimulus position with a frequency of $79 \%\left( \pm 8 \mathrm{SEM},(\mathrm{ScE} 1)_{6}\right.$-Gal4, ppk-eGFP /+) and 91\% ( \pm 5 SEM, UAS-mirr/+)). However, in ectopic mirr-expressing larvae only 56\% ( \pm 5 SEM) of rolls were initiated in the expected anticlockwise direction, suggesting that directional control over the escape response was lost (Fig. 7C). These data show that using ectopic Mirr activity to modify the sensory nervous system architecture increased sensitivity to noxious stimulation and altered directional control over the escape response.

\section{Discussion}

Current genetic models do not provide sufficient spatiotemporal precision to explain how specification of neuron modality, position, and topographic projections unify in a single developmental sequence. To address this issue, we used class IV nociceptive neurons as a powerful model in which to unambiguously identify every individual neural precursor, differentiating, and differentiated neuron. We find that class IV neurogenesis occurs through regionalized convergent programs that coordinate class-specification with axon projection topography.

\section{Regionalized controls over class IV neurogenesis}

In many neurogenetic contexts neurons of the same class arise from specialized developmental domains and fate control is the outcome of morphogen-mediated precursor patterning (O'Leary et al., 2007; Dasen, 2009; Erzurumlu et al., 2010). However, a dispersed organization of MD neuron classes in the body wall (Fig. 1A) may be necessary for correct sensory system activity, and it may not be easily achievable by classic morphogen action. Instead we find that it occurs through convergent class specification programs.

We first show that class IV patterning is an extension of the embryonic segmentation network. The potential to form class IV neurons is specified in the anterior epithelium of the parasegment by Odd, and repressed by in the posterior by Slp (Saulier-Le Dréan et al., 1998; Nasiadka and Krause, 2002). Odd, a transcriptional repressor (Goldstein et al., 2005), is upstream of class IV neurogenesis but is lost from the epithelium significantly before SOP1a and 4a formation. In both odd and slp mutant embryos alterations in MD fate occur following significant changes in parasegmental patterning. We suggest that direct transcriptional control of class IV neurogenesis by these factors is unlikely. In- 
stead, downstream of Odd and Slp, coordination between neuron position and modality is achieved by regionalized neurogenetic programs.

Close to the ventral midline, class IV neuron formation, but not fate, occurs through the Hh and Egfr pathways. Local class IV-specific differentiation is promoted by the prepatterning TF Mirr. Notably, disruption of $h h$, egfr, and mirr all give rise to incomplete phenotype penetrance possibly because vdaB specification programs are buffered by the combination of several pathways in a single position and short developmental period (Cooper and Albertson, 2008).

\section{Convergent neuronal class specification}

Although initially surprising, convergent class specification mechanisms may represent a more general phenomenon in nervous system development. Neuron classes are defined by shared properties, e.g., morphology and ion channel content. As such, neuron differentiation occurs by the concerted activity of a classspecific combination of genes, termed a gene-battery (Hobert et al., 2010). To create class-specific character, developmental programs must ensure that all the genes relevant to a specific class (i.e., all members of the gene-battery) are coexpressed. However, few, if any, members of a gene-battery are exclusive to a single neuron class and may be activated in multiple contexts. For example, in MD neurons $p p k$ is a hallmark class IV-specific ion channel. Nevertheless $p p k$ was recently found to also be expressed in adult reproductive tract neurons that monitor nutrient status and control mating behavior (Häsemeyer et al., 2009; Yang et al., 2009; Ribeiro and Dickson, 2010). A series of studies in Caenorhabditis elegans investigating the transcription control of genebattery members in neurons have found that expression can be regulated through more than one input pathway (Hobert et al., 2010). Here we show that even within the set of structural and functionally similar class IV neurons there are regional differences in the mechanisms that promote $p p k$ expression. Such findings are important because the existence of convergent neuron class specification mechanisms may prove useful when manufacturing unique neuron types for replacement therapies. Additional dissection of class IV neuron specification mechanisms may help clarify how convergence can be achieved.

\section{A genetic node linking neuron modality and topographic axonal projections}

A key finding of this study is that Mirr sets up a local prepattern to promote class IV character, and it also delineates the topographic axonal projections of vdaB. Hence Mirr acts as a genetic node linking regionalized neuron class specification to the control of axon projection topography.

As yet, the extent to which factors downstream of Mirr are shared between class specification and topography control programs remains unclear. We speculate the program controlling axon topography is significantly different from that inducing class IV-specific differentiation. Via a candidate approach, we identified $\mathrm{Kn}$ as one factor in the Mirr-controlled pathway. No role of $\mathrm{Kn}$ in axon topology control has been established; on the other hand $\mathrm{Kn}$ is central for the Mirr-mediated promotion of $p p k$ expression.

Mirr expression is lost before neuron differentiation. It may initiate a cascade of TFs culminating in the control of neuron differentiation. However, an intriguing alternative is that Mirr could directly control the expression of factors in precursor stages, and these factors are later used during neuron differentiation. There is increasing evidence that this kind of control over neuron differentiation can occur. For example, retinal neural precursors express neuron differentiation factors, and neurons derived from these precursors can differentiate within only 15 min of exiting the cell cycle (Livesey and Cepko, 2001). Moreover, within SOP lineages that give rise proprioceptive chordotonal sensory neurons, the basic helix-loop-helix TF Atonal is only expressed in precursor stages. However, Atonal drives expression of structural genes within the SOP and IPs that are used for structural differentiation of the proprioceptive neuron itself (Cachero et al., 2011).

Candidate and genetic screening approaches can be used to find more members of the Mirr-induced class specification and topography control programs. In addition, the rapid development of transcriptome profiling from a small number of cells along with TF-target identification techniques means that a genome-wide profiling approach to identify the factors downstream of Mirr can now be attempted. Utilizing genome-wide methods will help to elucidate the pathways by which cell fate convergence is achieved, and also the degree of linkage between class specification and topography control programs. Finally, ectopic Mirr in non-class IV lineages induces the formation of neurons with a class IV-identity dendritic repulsion program. Hence elucidating the programs downstream of this TF may provide a new route to identify factors acting in dendritic tiling (Grueber and Sagasti, 2010).

\section{Functional organization of the larval nociceptor-motoneuron circuit}

Drosophila is a potent model in which to study the genetic bases of behaviors (Vosshall, 2007). Activation of class IV neurons causes the larva to initiate a directional rolling response that has evolved as a nociceptive escape behavior (Hwang et al., 2007). Via ectopic expression of Mirr in non-class IV generating SOP lineages, we increased class IV neuron number. The sensory dendrites of these additional class IV neurons occupy the same broad regions of body wall as the endogenous class IV neuron. As such, a local stimulus is likely to activate an elevated number of nociceptive neurons, and notably we simultaneously found an increase in larval sensitivity to noxious-heat stimulation. In addition, although the larvae executed a rolling escape response with apparently normal muscle coordination, the directionality of rolling was uncorrelated with the direction of stimulation. As ectopic Mirr expression promotes ventral-like axon termini in the laterally situated v'ada, we speculate that these larvae are unable to correctly transmit the direction of stimulus to the escape circuit. It is also possible that activation of an elevated neuron number perturbs circuit activity in a way that disrupts directionality.

Motoneurons are the output of the neural circuit controlling the nociceptive escape behavior. Notably motoneuron dendrites form myotopic maps in the $\mathrm{VNC}$ that relate to the muscles they innervate in the body wall (Landgraf et al., 2003; Mauss et al., 2009). Nociceptor-motoneuron circuit activity may rely upon accurate matching of sensory axon and motoneuron dendrite mapping mechanisms. The rest of the circuit is as yet unknown; however, powerful genetic tools available in the fly can be used to further elucidate its neural substrates. Via genetic silencing or activation of subsets of neurons, it will be possible to identify neurons in the VNC that mediate escape behavior. It will also be possible to use genetic synapse mapping techniques such as GRASP (GFP Reconstitution Across Synaptic Partners) to detect the shared and differing direct synaptic partners in the VNC of class IV neurons situated in different regions of the body wall (Feinberg et al., 2008; Gordon and Scott, 2009). A further way to map neurons encoding escape directionality 
is via a direct readout of neural activity. Lateralized stimulation of class IV neurons (either electrical or optogenetic) coupled with a transgenic indicator of neuron activity can identify VNC neurons that show lateralized activation.

In this study we have demonstrated how unified genetic programs specify and position nociceptive neurons in the body wall and control their projections into the CNS. Future challenges are to elucidate the convergent genetic programs specifying sensory modality in the periphery, and also the programs translating body wall position into axonal projections. A further task is to map the nociceptor-motoneuron circuit in the VNC. It will then be possible to bring all these aspects together in a tractable model to investigate how developmental control over nervous system architecture shapes behavior.

\section{References}

Babcock DT, Landry C, Galko MJ (2009) Cytokine signaling mediates UV-induced nociceptive sensitization in Drosophila larvae. Curr Biol 19:799-806.

Buff E, Carmena A, Gisselbrecht S, Jiménez F, Michelson AM (1998) Signalling by the Drosophila epidermal growth factor receptor is required for the specification and diversification of embryonic muscle progenitors. Development 125:2075-2086.

Cachero S, Simpson TI, Zur Lage PI, Ma L, Newton FG, Holohan EE, Armstrong JD, Jarman AP (2011) The gene regulatory cascade linking proneural specification with differentiation in Drosophila sensory neurons. PLoS Biol 9:e1000568.

Cooper WJ, Albertson RC (2008) Quantification and variation in experimental studies of morphogenesis. Dev Biol 321:295-302.

Crozatier M, Vincent A (2008) Control of multidendritic neuron differentiation in Drosophila: the role of Collier. Dev Biol 315:232-242.

Dasen JS (2009) Transcriptional networks in the early development of sensory-motor circuits. Curr Top Dev Biol 87:119-148.

De Graeve F, Jagla T, Daponte JP, Rickert C, Dastugue B, Urban J, Jagla K (2004) The ladybird homeobox genes are essential for the specification of a subpopulation of neural cells. Dev Biol 270:122-134.

Erzurumlu RS, Murakami Y, Rijli FM (2010) Mapping the face in the somatosensory brainstem. Nat Rev Neurosci 11:252-263.

Feinberg EH, Vanhoven MK, Bendesky A, Wang G, Fetter RD, Shen K, Bargmann CI (2008) GFP Reconstitution Across Synaptic Partners (GRASP) defines cell contacts and synapses in living nervous systems. Neuron 57:353-363.

Goldstein RE, Cook O, Dinur T, Pisanté A, Karandikar UC, Bidwai A, Paroush Z (2005) An eh1-like motif in odd-skipped mediates recruitment of Groucho and repression in vivo. Mol Cell Biol 25:10711-10720.

Gordon MD, Scott K (2009) Motor control in a Drosophila taste circuit. Neuron 61:373-384.

Grueber WB, Sagasti A (2010) Self-avoidance and tiling: mechanisms of dendrite and axon spacing. Cold Spring Harb Perspect Biol 2:a001750.

Grueber WB, Jan LY, Jan YN (2003a) Different levels of the homeodomain protein cut regulate distinct dendrite branching patterns of Drosophila multidendritic neurons. Cell 112:805-818.

Grueber WB, Ye B, Moore AW, Jan LY, Jan YN (2003b) Dendrites of distinct classes of Drosophila sensory neurons show different capacities for homotypic repulsion. Curr Biol 13:618-626.

Grueber WB, Ye B, Yang CH, Younger S, Borden K, Jan LY, Jan YN (2007) Projections of Drosophila multidendritic neurons in the central nervous system: links with peripheral dendrite morphology. Development 134:55-64.

Hamada F, Tomoyasu Y, Takatsu Y, Nakamura M, Nagai S, Suzuki A, Fujita F, Shibuya H, Toyoshima K, Ueno N, Akiyama T (1999) Negative regulation of Wingless signaling by D-axin, a Drosophila homolog of axin. Science 283:1739-1742.

Häsemeyer M, Yapici N, Heberlein U, Dickson BJ (2009) Sensory neurons in the Drosophila genital tract regulate female reproductive behavior. Neuron 61:511-518.

Hattori Y, Sugimura K, Uemura T (2007) Selective expression of Knot/Collier, a transcriptional regulator of the EBF/Olf-1 family, endows the Drosophila sensory system with neuronal class-specific elaborated dendritic patterns. Genes Cells 12:1011-1022.

Hepker J, Wang QT, Motzny CK, Holmgren R, Orenic TV (1997) Drosophila cubitus interruptus forms a negative feedback loop with patched and regulates expression of Hedgehog target genes. Development 124:549-558.
Hobert O, Carrera I, Stefanakis N (2010) The molecular and gene regulatory signature of a neuron. Trends Neurosci 33:435-445.

Hwang RY, Zhong L, Xu Y, Johnson T, Zhang F, Deisseroth K, Tracey WD (2007) Nociceptive neurons protect Drosophila larvae from parasitoid wasps. Curr Biol 17:2105-2116.

Ingham PW, Hidalgo A (1993) Regulation of wingless transcription in the Drosophila embryo. Development 117:283-291.

Jinushi-Nakao S, Arvind R, Amikura R, Kinameri E, Liu AW, Moore AW (2007) Knot/Collier and cut control different aspects of dendrite cytoskeleton and synergize to define final arbor shape. Neuron 56:963-978.

Karim MR, Moore AW (2011) Morphological analysis of Drosophila larval peripheral sensory neuron dendrites and axons using genetic mosaics. J Vis Exp, in press

Landgraf M, Jeffrey V, Fujioka M, Jaynes JB, Bate M (2003) Embryonic origins of a motor system: motor dendrites form a myotopic map in Drosophila. PLoS Biol 1:E41.

Livesey FJ, Cepko CL (2001) Vertebrate neural cell-fate determination: lessons from the retina. Nat Rev Neurosci 2:109-118.

Long H, Ou Y, Rao Y, van Meyel DJ (2009) Dendrite branching and selfavoidance are controlled by Turtle, a conserved IgSF protein in Drosophila. Development 136:3475-3484.

Luo L, Flanagan JG (2007) Development of continuous and discrete neural maps. Neuron 56:284-300.

Mauss A, Tripodi M, Evers JF, Landgraf M (2009) Midline signalling systems direct the formation of a neural map by dendritic targeting in the Drosophila motor system. PLoS Biol 7:e1000200.

Moore AW, Jan LY, Jan YN (2002) hamlet, a binary genetic switch between singleand multiple-dendrite neuron morphology. Science 297:1355-1358.

Moore AW, Roegiers F, Jan LY, Jan YN (2004) Conversion of neurons and glia to external-cell fates in the external sensory organs of Drosophila hamlet mutants by a cousin-cousin cell-type respecification. Genes Dev 18:623-628.

Nasiadka A, Krause HM (2002) Transcriptional regulation during early Drosophila development. In: Advances in developmental biology and biochemistry (DePamphilis M, ed), pp 156-204. Burlington: Elsevier Science.

O'Leary DD, Chou SJ, Sahara S (2007) Area patterning of the mammalian cortex. Neuron 56:252-269.

Orgogozo V, Schweisguth F, Bellaïche Y (2001) Lineage, cell polarity and inscuteable function in the peripheral nervous system of the Drosophila embryo. Development 128:631-643.

Powell LM, Zur Lage PI, Prentice DR, Senthinathan B, Jarman AP (2004) The proneural proteins Atonal and Scute regulate neural target genes through different E-box binding sites. Mol Cell Biol 24:9517-9526.

Ribeiro C, Dickson BJ (2010) Sex peptide receptor and neuronal TOR/S6K signaling modulate nutrient balancing in Drosophila. Curr Biol 20:1000-1005.

Sanes JR, Zipursky SL (2010) Design principles of insect and vertebrate visual systems. Neuron 66:15-36.

Sanson B (2001) Generating patterns from fields of cells. Examples from Drosophila segmentation. EMBO Rep 2:1083-1088.

Saulier-Le Dréan B, Nasiadka A, Dong J, Krause HM (1998) Dynamic changes in the functions of Odd-skipped during early Drosophila embryogenesis. Development 125:4851-4861.

Schouenborg J (2002) Modular organisation and spinal somatosensory imprinting. Brain Res Brain Res Rev 40:80-91.

Soba P, Zhu S, Emoto K, Younger S, Yang SJ, Yu HH, Lee T, Jan LY, Jan YN (2007) Drosophila sensory neurons require Dscam for dendritic selfavoidance and proper dendritic field organization. Neuron 54:403-416.

Sugimura K, Yamamoto M, Niwa R, Satoh D, Goto S, Taniguchi M, Hayashi S, Uemura T (2003) Distinct developmental modes and lesion-induced reactions of dendrites of two classes of Drosophila sensory neurons. J Neurosci 23:3752-3760

Sugimura K, Satoh D, Estes P, Crews S, Uemura T (2004) Development of morphological diversity of dendrites in Drosophila by the BTB-zinc finger protein abrupt. Neuron 43:809-822.

Urbach R, Technau GM (2003) Molecular markers for identified neuroblasts in the developing brain of Drosophila. Development 130:3621-3637.

Urban S, Brown G, Freeman M (2004) EGF receptor signalling protects smooth-cuticle cells from apoptosis during Drosophila ventral epidermis development. Development 131:1835-1845.

Vosshall LB (2007) Into the mind of a fly. Nature 450:193-197.

Yang CH, Rumpf S, Xiang Y, Gordon MD, Song W, Jan LY, Jan YN (2009) Control of the postmating behavioral switch in Drosophila females by internal sensory neurons. Neuron 61:519-526. 